\title{
Propriedades termodinâmicas de adsorção de água do amido de rizomas do lírio-do-brejo (Hedychium coronarium)
}

\author{
Thermodynamic properties of water adsortion of the starch \\ of rhizome of swamp lily (Hedychium coronarium)
}

\author{
Diego Palmiro Ramirez ASCHERI ${ }^{1 *}$, Wellington de Souza MOURA², \\ José Luis Ramírez ASCHERI ${ }^{3}$, Edson Alves Freitas JUNIOR ${ }^{1}$
}

\section{Resumo}

Determinaram-se as propriedades termodinâmicas (entalpia diferencial, entropia diferencial, entalpia integral e entropia integral) do amido de rizomas do lírio-do-brejo (Hedychium coronarium) por meio de isotermas de adsorção de água. As isotermas foram determinadas em atividades de água no intervalo de 0,11 a 0,84 , sob temperaturas que variaram de 30 a $50{ }^{\circ} \mathrm{C}$. A Equação de GAB, que se ajustou bem às isotermas experimentais, foi utilizada para estimar as propriedades termodinâmicas de adsorção. As isotermas apresentaram ligeira inversão, indicando a precença de amido danificado. A entalpia diferencial e a entropia diferencial aumentaram com a diminuição da umidade de equilíbrio e correlacionaram entre si confirmando a compensação química linear. Um modelo exponencial do tipo $\mathrm{Y}=\mathrm{b}$. $\mathrm{e}^{\left(\mathrm{a} / \mathrm{X}_{\mathrm{e}}\right)}$ descreveu adequadamente a dependência destas propriedades diferenciais ao teor de umidade de equilíbrio. A entalpia integral e a entropia integral aumentaram continuamente com o teor de umidade de equilíbrio, porém com valores negativos para a entropia integral. Estas propriedades termodinâmicas de adsorção de água demonstraram que o amido extraído dos rizomas do lírio-do-brejo possui baixa higrocopicidade apesar da ocorrência de grânulos de amido danificados.

Palavras-chave: Hedychium coronarium; isotermas de adsorção de água; calor isostérico; atividade de água; fécula.

\begin{abstract}
Thermodynamic properties where determined (differential enthalpy, of differential entropy, integral enthalpy, and integral entropy) of the starch of rhizomes of swamp lily through water adsorption isotherms. The isotherms were determined in water activities in the range of 0.11 to 0.84 , in temperatures ranging from 30 to $50^{\circ} \mathrm{C}$. The $\mathrm{GAB}$ equation which fits well to the experimental isotherms was used to estimate the properties of thermodynamic adsorption. The slight reversal of the isotherms indicates damaged starch. The enthalpy and entropy differential gap increased with the decrease in moisture and correlated to each other confirming the chemical linear compensation. An exponential model type $\mathrm{Y}=\mathrm{b} \cdot \mathrm{e}^{\left(\mathrm{a} / \mathrm{x}_{\mathrm{e}}\right)}$ described adequately the dependence of these properties differential on the moisture content of balance. The integral enthalpy and entropy increased continuously with the moisture content of balance but presented negative values for the integral entropy. These thermodynamic properties of water adsorption showed that the starch extracted from the rhizomes of swamp lily has low hygroscopicity despite the occurrence of damaged starch granules.
\end{abstract}

Keywords: Hedychium coronarium; water adsorption isotherm; isosteric heat; water activity; starch.

\section{Introdução}

O lírio-do-brejo é uma macrófita aquática de ampla distribuição geográfica que vai desde o Himalaia até a China na Ásia tropical e em todas as Américas desde os Estados Unidos até a Argentina. Foi introduzido no Brasil em 1987, disseminando pelo País, sendo mais comum em toda a zona litorânea. Denominado cientificamente de Hedychium coronarium, o lírio-do-brejo pertence à ordem Scitaminae da família Zingiberaciae e recebe outros nomes comuns como jasmim-do-brejo, são-josé e gengibrebranco (KISSMANN; GROTH, 1991; MACEDO, 1997).

No Brasil, o lírio-do-brejo é considerado uma planta exótica invasora e atualmente vêm se estudando o manejo e o controle desta praga, uma vez que a conseqüência negativa sobre as espécies nativas torna esta planta daninha nos locais onde prolifera (SANTOS; PEDRALLI; MEYER, 2005).

Apresenta uma ampla utilização, principalmente farmacológica e ornamental (AGAREZ; PEREIRA; ATIDIO, 1994), no entanto, apesar do teor de amido nos rizomas do lírio-do-brejo, não há na literatura estudos sobre este amido natural, tornando-se necessárias pesquisas sobre suas propriedades termodinâmicas de adsorção de água aplicadas ao processo de secagem do amido.

As propriedades termodinâmicas de adsorção de água são fundamentais para a análise de projetos de equipamentos em

Recebido para publicação em 29/1/2008

Aceito para publicação em 3/1/2009 (003184)

1 Universidade Estadual de Goiás - UEG, BR 153, km 98, CEP 75001-970, Anápolis, Goiás, Brasil, E-mail: ascheridpr@uol.com.br

${ }^{2}$ Programa de Mestrado de Ciências Moleculares, Universidade Estadual de Goiás-UEG, BR 153, km 98, CEP 75001-970, Anápolis, Goiás, Brasil,

E-mail: bussund@gmail.com

${ }^{3}$ Embrapa Agroindústria de Alimentos, Av. das Américas 29501, Guaratiba, CEP 23020-470, Rio de Janeiro, RJ, Brasil, E-mail: ascheri@ctaa.embrapa.br

${ }^{*}$ A quem a correspondência deve ser enviada 
vários processos de preservação, secagem, armazenamento, acondicionamento e misturas de alimentos (IGLESIAS; CHIRIFE; VIOLLAZ, 1976; AVIARA; AJIBOLA; ONI, 2004; KAYA; KAHYAOGLU, 2006).

Estas propriedades podem ser usadas para estimar a mínima quantidade de energia requerida na desidratação e fornece informações a respeito do estado da água no produto alimentício. Também, as determinações destas propriedades são necessárias, quando a taxa de sorção de água (absorção ou desorção) é dada em função de um processo simultâneo de transferência de calor e massa, para predizer parâmetros da cinética do fenômeno de sorção e fazer deduções aproximadas da microestrutura e das mudanças físicas que ocorrem na superfície do produto alimentício (AGUERRE; SUAREZ; VIOLLAZ, 1986).

De acordo com Kaya, Kahyaoglu (2006), as propriedades termodinâmicas são calculadas através de isotermas de sorção e as principais funções são aquelas que quantificam as entalpias e entropias diferenciais e integrais fundamentais na análise da exigência energética e predição dos parâmetros cinéticos nos processos de sorção.

O calor diferencial de sorção $\left(\Delta H_{d}\right)$ é usado como um indicador do estado em que se encontra a água presente num material biológico (FASINA; SOKHANSANJ; TYLER, 1997), de acordo com o tipo de força excida na interligação molecular do vapor de água com os sítios de sorção.

$\mathrm{O} \Delta \mathrm{H}_{\mathrm{d}}$ é utilizada para projetar secadores capazes de fornecer calor acima do calor latente de vaporização da água pura $\left(\lambda_{\mathrm{vap}}\right)$ de modo a secar o material em baixos níveis de teor de umidade. É calculada em base ao calor líquido de sorção $\left(\mathrm{q}_{\mathrm{st}}\right)$ definido como a diferença entre $\Delta \mathrm{H}_{\mathrm{d}}$ e $\lambda_{\text {vap }}$, para uma determinada temperatura (TSAMI et al., 1990). O q st $_{\text {st }}$ se origina da Equação de Clausius-Clapeyron assumindo que o calor latente de vaporização da água pura, o calor líquido de sorção e a umidade de equilibrio permanecem constantes com a variação da temperatura.

A entropia diferencial $\left(\Delta \mathrm{S}_{\mathrm{d}}\right)$ relaciona-se com o número de sítios de sorção para um determinado nível de energia inerente ao material biológico (MADAMBA; DRISCOLL; BUCKLE, 1996). A $\Delta \mathrm{S}_{\mathrm{d}}$ pode ser usada para obter informações quanto à racionalização da energia durante o processamento, dissolução e cristalização do produto (AVIARA; AJIBOLA; DAIRO, 2002).

As quantidades integrais permitem a interpretação qualitativa do fenômeno de sorção. Descrevem o grau de desordem e o movimento desordenado das moléculas de água, além da energia envolvida nos processos de sorção. Estas funções derivam-se da primeira lei da termodinâmica aplicada para o material adsorvente puro (RIZVI, 1986).

A entalpia integral líquida de sorção $\left(\mathrm{q}_{\mathrm{eq}}\right)$ é calculada de forma semelhante ao cálculo de $\Delta \mathrm{H}_{\mathrm{d}}$, porém fazendo uso de uma nova constante chamada de spreading pressure $(\phi)$ ao invés da $\mathrm{X}_{\mathrm{e}}$ (BENADO; RIZVI, 1985). O $\phi$ é definido como a energia responsável pela difusão da água pelos poros do material durante o processo de sorção e depende da temperatura e da atividade de água $\left(\mathrm{A}_{\mathrm{w}}\right)$, sendo usado na interpretação da cinética de sorção.
Stamm (1964) referiu-se ao $\phi$ como a energia livre da superfície de sorção, podendo ser estimado através da diferença entre o potencial de superfície dos sítios de sorção livres e das moléculas sorvidas.

No presente trabalho se determinaram as propriedades termodinâmicas (entalpia diferencial, entropia diferencial, entalpia integral e entropia integral) de amido extraído dos rizomas do lírio-do-brejo por meio de isotermas de adsorção de água em atividades de água que variaram de 0,11 a 0,84 , em temperaturas de 30,40 e $50{ }^{\circ} \mathrm{C}$.

\section{Material e métodos}

\subsection{Matéria-prima}

Os rizomas do lírio-do-brejo (H. coronarium) foram obtidos de plantas sadias das margens do córrego Restinga da fazenda Mata Taquaral (Orizona, GO), colhidas em fevereiro de 2007. O clima da região é tropical úmido, caracterizado por duas estações bem definidas: a seca, quando a precipitação média fica abaixo de 10 mm mensais; e a estação úmida, com média mensal acima de $250 \mathrm{~mm}$, com uma temperatura média de $22^{\circ} \mathrm{C}$. A latitude é de $17^{\circ} 1^{\prime} 51^{\prime}$ ' S, a longitude de $48^{\circ} 17^{\prime} 45^{\prime \prime} \mathrm{O}$ e a altitude de $1000 \mathrm{~m}$ acima do nível do mar.

\subsection{Preparo das amostras}

Os rizomas foram selecionados de acordo com o estado de maturação, sendo estes reconhecidos pela cor amarelada quando fisiologicamente desenvolvidos (maduros), lavados com água corrente, arejados e transportados em sacos de polietileno para minimizar perdas de umidade até seu posterior uso.

Para a obtenção do amido os rizomas foram descascados, fazendo uso de facas de aço inoxidável, picados e recebidos em baldes plásticos contendo uma solução de metabissulfito $\left(5 \mathrm{~g} . \mathrm{L}^{-1} \mathrm{de}\right.$ água), numa proporção de $1 \mathrm{~kg}$ de matéria-prima/2 L de solução de metabissulfito. A moagem foi feita fazendo uso de um moinho de facas tipo "CROTON", modelo MA580 (MARCONI, Piracicaba, Brasil) com malha de 10 mesh, obtendo-se uma massa. Esta foi passada em peneiras de 100 e 325 mesh, com sucessivas lavagens com água corrente para separação do amido e posterior decantação em bacias plásticas. $\mathrm{O}$ material decantando foi lavado com álcool absoluto, filtrado a vácuo e desidratado a $75^{\circ} \mathrm{C}$ por 12 horas. $\mathrm{O}$ amido desidratado permaneceu 12 horas num dessecador contendo sílica-gel como agente dessecante e em seguida foi reduzido a pó utilizando-se gral e pistilo e peneirado em peneira de 48 mesh, obtendo-se assim a amostra seca para análise.

Foram tomadas microfotografias do amido isolado para análise visual de amido danificado. Para este fim, amostras de amido foram coletadas com fio de platina e misturadas, sobre lâmina de vidro, com uma gota de lugol e, posteriormente, cobertas com lamínula. As lâminas foram observadas em microscópio óptico LEICA (DME, Wetzlar, Alemanha) e as imagens selecionadas foram analisadas pelo software LAS EZ.

\subsection{Determinação da composição centesimal}

$\mathrm{O}$ amido foi caracterizado quanto ao $\mathrm{pH}$, acidez titulável, umidade, cinzas, amido, açúcares totais, fibras, matéria graxa 
e proteína (AOAC, 2000). O teor de amilose foi determinado segundo a metodologia de Williams, Kuzina, Hlynka (1970).

\subsection{Determinação das isotermas de adsorção}

As isotermas foram determinadas de acordo com o Projeto COST 90 (ASCHERI; NASCIMENTO; ASCHERI, 2003). As soluções saturadas utilizadas e respectivas atividades de água geradas em cada uma das temperaturas de 30,40 e $50^{\circ} \mathrm{C}$ estão na Tabela 2.

A determinação da massa seca $\left(\mathrm{m}_{\mathrm{i}}\right)$ foi realizada colocandose aproximadamente $0,5 \mathrm{~g}$ da amostra em pesa-filtro de $25 \mathrm{~mL}$, previamente tarado e transferido para a estufa a $75{ }^{\circ} \mathrm{C}$ por aproximadamente 4 horas, segundo o método da AOAC (2000). Em seguida, os pesa-filtros foram colocados, sobre suporte, nos dessecadores contendo as soluções saturadas. O processo foi acompanhado até não haver mais variação de massa $\left(\mathrm{m}_{\mathrm{e}}\right)$. Os valores de umidade de equilíbrio $\left(\mathrm{X}_{\mathrm{e}}\right)$ foram calculados através da Equação 1:

$X_{e}=\frac{m_{e}-m_{i}}{m_{i}} \times 100$

A Equação de GAB (Guggenheim-Anderson-de Boer) foi usada para analisar os dados das isotermas de adsorção de água Equação 2:

$X_{e}=\frac{X_{m} \cdot C \cdot k \cdot A_{w}}{\left(1-k \cdot A_{w}\right) \cdot\left(1-k \cdot A_{w}+C \cdot k \cdot A_{w}\right)}$

em que:

$\mathrm{X}_{\mathrm{m}}=$ umidade na monocamada quantificada em $\mathrm{kg}$ água $/ 100 \mathrm{~kg}$ de m. s.;

$\mathrm{C}=$ constante de Guggenheim relacionada às propriedades da monocamada; e

$\mathrm{k}=$ fator que corresponde às propriedades da multicamada.

\subsection{Propriedades termodinâmicas}

O calor diferencial de sorção $\left(\Delta \mathrm{H}_{\mathrm{d}}\right), \mathrm{em} \mathrm{kJ} / \mathrm{mol}$, foi determinado utilizando-se a Equação 3:

$\Delta H_{d}=q_{s t}+\lambda_{\text {vap }}$

O calor latente de vaporização da água pura $\left(\lambda_{\text {vap }}\right)$, na temperatura máxima de trabalho $\left(50^{\circ} \mathrm{C}\right)$, o qual é $43,2 \mathrm{~kJ} / \mathrm{mol}$, foi obtido a partir da Equação (4), com $\mathrm{T}$ em graus Celsius (MESSE, 2007) Equação 4:

$\lambda_{\text {vap }}=44,72-0,03 T-9,2.10^{-5} T^{2} ; R^{2}=0,999$

O calor líquido de sorção $\left(\mathrm{q}_{\mathrm{st}}\right)$, em $\mathrm{kJ} / \mathrm{mol}$, foi calculado utilizando-se a forma integrada da Equação de Clausius-Clapeyron relacionando a atividade de água e a temperatura absoluta (em graus Kelvin, $\mathrm{K}$ ) correspondentes a uma determinada umidade de equilíbrio (RIZVI, 1986; BELL; LABUZA, 2000) Equação 5: $\left[\ln \left(\frac{A_{w_{1}}}{A_{w_{2}}}\right)=\frac{-q_{s t}}{R} \cdot\left(\frac{1}{T_{1}}-\frac{1}{T_{2}}\right)\right]_{X_{e}}$

em que:

$\mathrm{R}=$ constante real dos gases igual a $0,008314 \mathrm{~kJ} / \mathrm{mol}-\mathrm{K}$.

Para este cálculo assume-se que o calor de sorção seja independente das mudanças de temperatura. O coeficiente angular da reta $\ln \left(\mathrm{A}_{\mathrm{w}}\right)$ versus $1 / \mathrm{T}$ para $\mathrm{X}_{\mathrm{e}}$ constante corresponde ao valor do calor líquido de sorção (Equação 5).

A entropia diferencial $\left(\Delta \mathrm{S}_{\mathrm{d}}\right)$, em $\mathrm{kJ} / \mathrm{mol}-\mathrm{K}$, foi determinada (MCMINN; MAGEE, 2003; KAYA; KAHYAOGLU, 2005) utilizando-se a Equação 6:

$\Delta S_{d}=\frac{\Delta H_{d}-\Delta G}{T}$

A energia livre de Gibbs $(\Delta \mathrm{G})$, em $\mathrm{kJ} / \mathrm{mol}$, foi calculada a partir da Equação 7:

$\Delta G=R \cdot T \cdot \ln \left(A_{w}\right)$

A entalpia integral líquida de sorção $\left(\mathrm{q}_{\mathrm{eq}}\right)$, em $\mathrm{kJ} / \mathrm{mol}$, foi obtida de forma semelhante ao cálculo do calor líquido de sorção (Equação 5). A Equação de $\mathrm{q}_{\mathrm{eq}}$ baseada na Equação de Gibbs (HILL; RIZVI, 1982) para valores de Spreading Pressure constantes é Equação 8:

$\left[\ln \left(\frac{A_{w_{1}}}{A_{w_{2}}}\right)=\frac{-q_{e q}}{R} \cdot\left(\frac{1}{T_{1}}-\frac{1}{T_{2}}\right)\right]_{\phi}$

O cálculo do Spreading Pressure ( $\phi, \mathrm{em} \mathrm{kJ} / \mathrm{m}^{2}$ ) foi determinado de acordo com o procedimento analítico descrito por Iglesias, Chirife, Viollaz (1976) e Fasina, Ajibola, Tyler (1999), mostrado na Equação 9:

$\phi=\frac{K_{B} T}{A_{m}} \cdot \int_{0}^{A_{w}} \frac{X_{e}}{X_{m} A_{w}} \cdot d A_{w}$

em que:

$\mathrm{K}_{\mathrm{B}}=$ constante de Boltzmann $\left(1,380 \times 10^{-23} \mathrm{~J} / \mathrm{K}\right)$; e

$\mathrm{A}_{\mathrm{m}}=$ área superficial de uma molécula de água igual a $1,06 \times 10^{-19} \mathrm{~m}^{2}($ MAZZA,1980).

Quando a $A_{w}$ tende a zero, a integral da Equação 9 é indeterminada $\left(\ln \mathrm{A}_{\mathrm{w}} \rightarrow \infty\right)$, por isso, para o cálculo da integral desta Equação se deve assumir uma relação empírica entre a atividade de água e seu respectivo valor de umidade de equilíbrio (LI et al., 1994). O Spreading Pressure, para as diferentes temperaturas estudadas, foi calculado em valores de $A_{w} \geq 0,05$ utilizando-se a Equação de GAB (Equação 2), como segue na Equação 10:

$\phi=\frac{K_{B} T}{A_{m}} \cdot \ln \left[\frac{1+C \cdot k \cdot A_{w}-k \cdot A_{w}}{1-k \cdot A_{w}}\right]_{0,05}^{A_{w}}$

A entropia integral total quantificada em $\mathrm{kJ} / \mathrm{mol}-\mathrm{K}$ foi calculada como (BENADO; RIZVI, 1985) Equação 11: 
$\Delta S_{e q}=-\frac{q_{e q}}{T}-R \cdot \ln \left(A_{w}^{*}\right)$

em que:

$\mathrm{A}_{\mathrm{W}}^{*}=$ média geométrica de $\mathrm{A}_{\mathrm{w}}$, calculada de acordo com a Equação 12:

$A_{w}^{*}=\left[\left(A_{w}\right)_{\phi_{30}{ }^{\circ} \mathrm{C}} \cdot\left(A_{w}\right)_{\phi_{40}{ }^{\circ} \mathrm{C}} \cdot\left(A_{w}\right)_{\phi_{50}{ }^{\circ} \mathrm{C}}\right]^{l}$

Os termos da direita da Equação 13 são calculados explicitamente substituindo $\mathrm{X}_{\mathrm{e}}$ da Equação 2 por $\phi$, ficando como:

$\left(A_{w}\right)_{\varphi_{T \rho C}}=\left[\frac{2+\left(X_{m} / \varphi-1\right) C-\left\{\left[2+\left(X_{m} / \varphi-1\right) C\right]^{2}-4(1-C)\right\}^{0,5}}{2 k(1-C)}\right]_{T \rho C}$

A teoria da compensação foi analisada em base à correlação linear entre os dados de $\Delta H_{d}$ versus $\Delta S_{d}$ de acordo com a Equação 14:

$\Delta H_{d}=T_{F} \cdot \Delta S+\Delta G$

em que:

$\mathrm{T}_{\mathrm{F}}=$ temperatura isocinética (em graus Kelvin), que foi comparada com a média harmônica das temperaturas utilizadas na experimentação;

$\mathrm{T}_{\mathrm{hm}}=$ temperatura média harmônica, calculada de acordo com a Equação 15:

$$
T_{h m}=\frac{3}{\sum_{i=1}^{3} \frac{1}{T_{i}}}
$$

em que:

$\mathrm{T}_{\mathrm{i}}=$ temperatura absoluta de 30,40 e $50^{\circ} \mathrm{C}$.

\subsection{Análise estatística dos resultados}

Os valores calculados das propriedades termodinâmicas em função da umidade de equilíbrio, quando $\mathrm{X}_{\mathrm{e}} \geq 5,0 \mathrm{~kg}$ de água $/ \mathrm{kg}$ de matéria seca, e os valores experimentais das isotermas de adsorção foram analisados através de regressão não linear ao nível de 95\% de confiança (GOMES, 2000), aplicando uma Equação exponencial (Equação 16), para as propriedades termodinâmicas, e a Equação de GAB (Equação 2), para as isotermas.

$Y=Y_{0}+a \cdot e^{\left(b / X_{e}\right)}$

em que:

$\mathrm{Y}=$ variável dependente $\left(\Delta \mathrm{H}_{\mathrm{d}}, \Delta \mathrm{S}_{\mathrm{d}}\right)$;

$\mathrm{Y}_{0}=$ valor inicial da variável dependente; $\mathrm{e}$

a e b = coeficientes da Equação 16.

A correlação entre a entalpia e entropia diferenciais foi obtida através de regressão linear ao nível de 95\% de confiança, de acordo com a Equação 14.

Foi estimado o coeficiente de determinação $\left(\mathrm{R}^{2}\right)$ e aplicado o teste qui-quadrado $\left(\chi^{2}\right)$ para verificar a adEquação do ajuste das Equações 2, 14 e 16 aos dados experimentais. Os coeficientes dessas equações e os respectivos valores de $\mathrm{R}^{2}$ e $\chi^{2}$ foram estimados com a ajuda do microcalculador Origin 5.0.

\section{Resultados e discussão}

\subsection{Composição centesimal do amido}

A Tabela 1 mostra que a purificação do amido foi bem sucedida uma vez que o amido possui teores em sua composição química de acordo com os estabelecidos pela Legislação Brasileira (BRASIL, 1978) para amidos comerciais.

O teor de amilose foi de 37,2\%. Comparando-se este valor com os da literatura, observou-se que é maior do que o de raízes e tubérculos como gengibre (Zingiber officinale) (REYES et al., 1982), mandioca (Manihot esculenta) (HOOVER, 2001) e biri (Canna edulis) (LEONEL et al., 2002), de sementes como Amaranto (Amaranthus cruentus) (HOOVER; SINNOTT; PERERA, 1998), castanha (Castanea sativa, Mill) (DEMIATE; OETTERER; WOSIACKI, 2001), arroz (Oriza sativa) (VERWIMP et al., 2004) e grão-de-bico (Cicer arietinum L.) (SINGH et al., 2004) que foram de 22,2, 18,6 a 28,6 e 33,0 e de, 3,5 a 4,8, 21,5, 29,7 e de 28,6 a $34,3 \%$, respectivamente.

\subsection{Isotermas de adsorção de água}

Os dados experimentais obtidos por meio das isotermas de adsorção às temperaturas de 30 a $50{ }^{\circ} \mathrm{C}$ para o amido dos rizomas do lírio-do-brejo estão na Tabela 2.

Na Tabela 3 têm-se os valores dos parâmetros estimados, obtidos por regressão não linear, da Equação de GAB, para o ajuste das isotermas de amido extraído dos rizomas do lírio-do-brejo, para cada temperatura estudada, assim como os coeficientes de determinação $\left(\mathrm{R}^{2}\right)$ e qui-quadrado $\left(\chi^{2}\right)$.

A partir dos valores do coeficiente de determinação e do qui-quadrado apresentados na Tabela 3, verifica-se que a Equação de GAB se ajustou bem aos dados experimentais das isotermas de adsorção obtidas das diferentes amostras estudadas.

Os coeficientes de determinação apresentaram valores próximos à unidade, explicando que o $99 \%$ da variação total da umidade de equilíbrio do amido em estudo encontram-se em torno da média e menos de $1 \%$ desta variação atribui-se aos

Tabela 1. Composição química do amido de rizomas do lírio-do-brejo (H. coronarium) em porcentagem em base úmida.

\begin{tabular}{lc}
\hline \multicolumn{1}{c}{ Análises } & Composição química \\
\hline Umidade & $6,95 \pm 0,04$ \\
Amido & $87,25 \pm 1,00$ \\
Cinzas & $0,50 \pm 0,02$ \\
Matéria graxa & $0,01 \pm 0,01$ \\
Fibras & $0,24 \pm 0,02$ \\
Proteínas & $0,03 \pm 0,01$ \\
Açúcares totais & $0,05 \pm 0,01$ \\
Amilose & $37,20 \pm 1,35$ \\
pH & $6,37 \pm 0,01$ \\
Acidez titulável $(\mathrm{mL} \mathrm{NaOH} 1 \mathrm{~N})$ & $0,73 \pm 0,06$ \\
\hline
\end{tabular}


erros experimentais. O teste $\chi^{2}$ aplicado demonstrou que os dados observados das isotermas e os dados estimados pela Equação de GAB se comportam de forma semelhante e a diferença entre estes são muito pequenas, próximas a zero, ao nível de 95\% de confiança $(\mathrm{p}<0,05)$.

A Figura 1 mostra o valor de $\mathrm{X}_{\mathrm{e}}$ em relação à $\mathrm{A}_{\mathrm{w}}$ para o amido de rizomas do lírio-do-brejo. Em valores constantes de $\mathrm{A}_{\mathrm{w}}<0,5$ observa-se diminuição de $\mathrm{X}_{\mathrm{e}}$ com o aumento da temperatura. Este fato se deve ao aumento do grau de desordem molecular da água adsorvida na superfície do amido pelo que se torna instável com o aumento da temperatura, facilitando a quebra das ligações intermoleculares entre as moléculas de água e os sítios de adsorção presentes no amido (HOSSAIN et al., 2001). Entretanto, acima destes valores se observa interseção entre as isotermas, observando-se maior o valor de $\mathrm{X}_{\mathrm{e}}$ quanto maior a temperatura de adsorção, provavelmente devido à presença de grânulos de amido danificados (Figura 2) causados por fatores inerentes ao processo de extração e purificação do amido, tornando-as, de certo modo, solúveis em $\mathrm{A}_{\mathrm{w}}>0,5$. Também pode ser que as temperaturas de 40 e $50{ }^{\circ} \mathrm{C}$ utilizadas para esta análise e em presença de atividade de água elevada intumesçam os grânulos de amido que podem se quebrar dando lugar a grupos hidroxilas $(-\mathrm{OH})$ livres das cadeias de amilose e de amilopectina, aumentando os sítios de adsorção de água.

\subsection{Entalpia e entropia diferenciais de adsorção}

Pela Figura 3 observa-se aumento de $\Delta \mathrm{H}_{d}$ com a diminuição de $\mathrm{X}_{\mathrm{e}}$, indicando a quantidade de energia requerida para remover moléculas de água ligadas à estrutura do amido em baixos teores de $\mathrm{X}_{\mathrm{e}}$. Na fase inicial de adsorção, na superfície do amido existem sítios de adsorção altamente polares nos quais as moléculas de água estão fortemente ligadas em forma de monocamada. Aqui, a energia de interação molécula de água/ amido é maior que a energia de interação entre moléculas de água pura $\left(\lambda_{\text {vap }}=43,35 \mathrm{~kJ} / \mathrm{mol}\right.$ ) (AL-MUHTASEB; MCMINN; MAGEE, 2004; ROMERO et al., 2005; FASINA; 2006).

Quando o nível de umidade de equilíbrio aumenta, a disponibilidade dos sítios de adsorção de água altamente polares diminui pelo que se observa diminuição no valor de $\Delta \mathrm{H}_{\mathrm{d}}$ até valores próximos ao valor do calor latente de vaporização da

Tabela 2. Valores de umidade de equilíbrio $\left(\mathrm{X}_{\mathrm{e}}\right)$ e atividades de água $\left(\mathrm{A}_{\mathrm{w}}\right)$ de distintos sais, de amido de rizomas de lírio-do-brejo (Hedychium coronarium).

\begin{tabular}{lrrrrrr}
\hline \multicolumn{1}{c}{ Sal } & \multicolumn{2}{c}{$30^{\circ} \mathrm{C}$} & \multicolumn{2}{c}{$40^{\circ} \mathrm{C}$} & \multicolumn{2}{c}{$50^{\circ} \mathrm{C}$} \\
\cline { 2 - 7 } & \multicolumn{1}{c}{$\mathrm{A}_{\mathrm{w}}$} & \multicolumn{1}{c}{$\mathrm{X}_{\mathrm{e}}$} & \multicolumn{1}{c}{$\mathrm{A}_{\mathrm{w}}$} & \multicolumn{1}{c}{$\mathrm{X}_{\mathrm{e}}$} & \multicolumn{1}{c}{$\mathrm{A}_{\mathrm{w}}$} & \multicolumn{1}{c}{$\mathrm{X}_{\mathrm{e}}$} \\
\hline $\mathrm{LiCl}$ & 0,113 & 4,777 & 0,112 & 4,321 & 0,111 & 3,712 \\
$\mathrm{CH}_{3} \mathrm{COOK}$ & 0,216 & 6,598 & 0,204 & 5,918 & 0,192 & 5,224 \\
$\mathrm{MgCl}_{2}$ & 0,324 & 8,151 & 0,316 & 7,730 & 0,305 & 7,242 \\
$\mathrm{~K}_{2} \mathrm{CO}_{3}$ & 0,432 & 9,910 & 0,432 & 9,775 & 0,433 & 9,585 \\
$\mathrm{Mg}\left(\mathrm{NO}_{3}\right)_{2}$ & 0,514 & 11,455 & 0,484 & 10,764 & 0,454 & 10,005 \\
$\mathrm{KI}$ & 0,679 & 15,288 & 0,661 & 15,207 & 0,645 & 15,098 \\
$\mathrm{NaCl}$ & 0,751 & 17,537 & 0,747 & 17,889 & 0,744 & 19,176 \\
$\mathrm{KCl}$ & 0,836 & 22,391 & 0,823 & 22,844 & 0,812 & 23,767 \\
\hline
\end{tabular}

Fonte: Greenspan (1977) e Palipane, Driscoll (1992). água pura, fato que indica a dissolução endotérmica do amido pela presença de grânulos danificados (AVIARA; AJIBOLA; DAIRO, 2002; KAYA; KAHYAOGLU, 2006), uma vez que aumenta os níves de umidade em baixos níveis de energia.

A $\mathrm{X}_{\mathrm{e}}$ em que $\Delta \mathrm{H}_{\mathrm{d}}$ aproxima-se a $\lambda_{\text {vap }}$ foi de $11 \mathrm{Kg}$ de água por Kg de matéria seca.

Confrontado com os resultados obtidos da literatura, o amido de rizomas do lírio-do-brejo mostra baixo valor de $\Delta \mathrm{H}_{\mathrm{d}}$ (aproximadamente $58 \mathrm{~kJ} / \mathrm{mol}$ ) em menores valores de $\mathrm{X}_{\mathrm{e}} \mathrm{em}$ comparação com o amido de batata, amido com alto teor de amilose e amido com alto teor de amilopectina (AL-MUHTASEB; MCMINN; MAGEE, 2004), amido de pupunha (FERREIRA; PENA, 2003) e polpa de manga (ROMERO et al., 2005) que apresentaram valor de $\Delta \mathrm{H}_{\mathrm{d}}$ próximos de $80 \mathrm{~kJ} / \mathrm{mol}$. Este fato sugere que o amido de rizomas do lírio-do-brejo possui

Tabela 3. Parâmetros da Equação de GAB aplicada às isotermas de adsorção do amido de rizomas do lírio-do-brejo, coeficientes de determinação $\left(\mathrm{R}^{2}\right)$ e qui-quadrado $\left(\chi^{2}\right)$, nas temperaturas de 30,40 e $50^{\circ} \mathrm{C}$.

\begin{tabular}{ccrccc}
\hline Temperatura & \multicolumn{5}{c}{ Parâmetro } \\
\hline$\left({ }^{\circ} \mathrm{C}\right)$ & $\mathrm{X}_{\mathrm{m}}$ & \multicolumn{1}{c}{$\mathrm{C}$} & $\mathrm{k}$ & $\mathrm{R}^{2}$ & $\chi^{2}$ \\
\hline 30 & 7,000 & 15,974 & 0,829 & 0,999 & $4,098 \cdot 10^{-6}$ \\
40 & 6,977 & 11,864 & 0,853 & 0,999 & $7,151 \cdot 10^{-6}$ \\
50 & 7,124 & 7,994 & 0,879 & 0,999 & $8,008 \cdot 10^{-7}$ \\
\hline
\end{tabular}

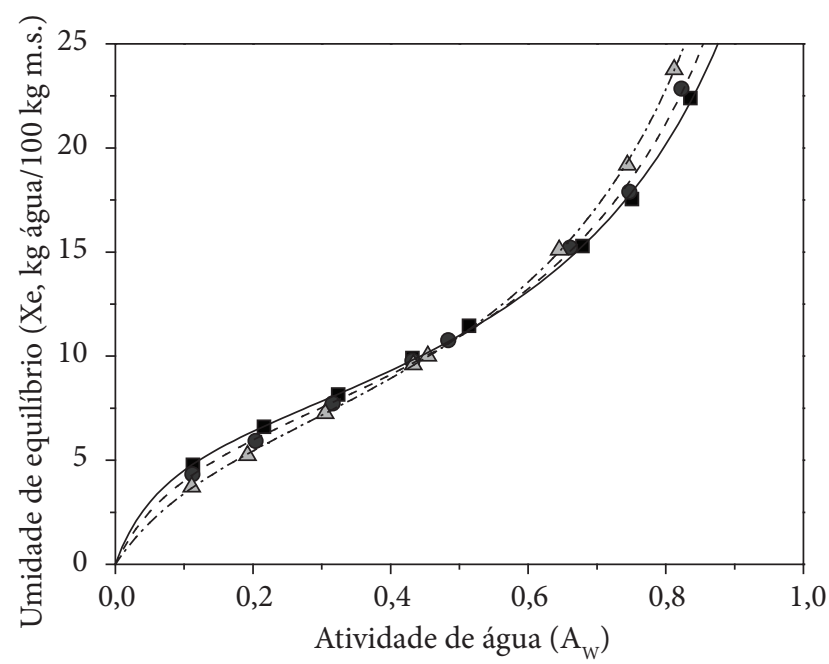

Dados experimentais

- à $30^{\circ} \mathrm{C}$

- à $40^{\circ} \mathrm{C}$

$\triangle$ à $50^{\circ} \mathrm{C}$

Dados ajustados ao modelo de GAB

$-X_{e}=92,759 \cdot A_{w} /\left(\left(1-0,829 \cdot A_{w}\right) \cdot\left(1-0,829 \cdot A_{w}+13,252 \cdot A_{w}\right)\right)$

- - $X_{e}=70,658 \cdot A_{w} /\left(\left(1-0,854 \cdot A_{w}\right) \cdot\left(1-0,854 \cdot A_{w}+10,127 \cdot A_{w}\right)\right)$

$\cdots \quad X_{e}=50,075 \cdot A_{w} /\left(\left(1-0,879 \cdot A_{w}\right) \cdot\left(1-0,879 \cdot A_{w}+7,029 \cdot A_{w}\right)\right)$

Figura 1. Isotermas de adsorção do amido de rizomas do lírio-do-brejo (H. coronarium). 


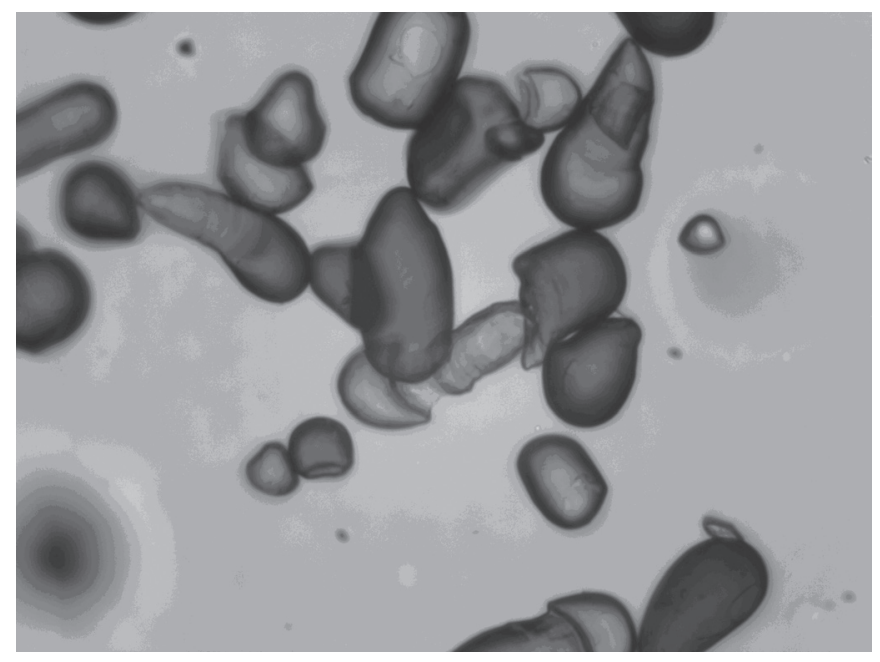

Figura 2. Microfotografia de amido de rizomas do lírio-do-brejo (H. coronarium) mostrando grânulos inteiros e danificados.

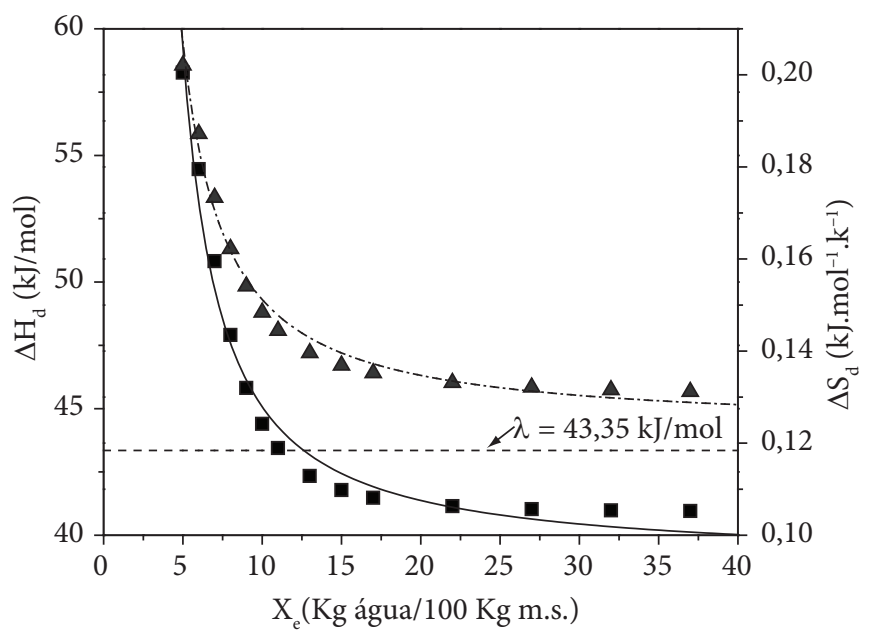

$$
\begin{aligned}
& \text { Dados experimentais } \\
& \text { - } \Delta \mathrm{H}_{\mathrm{d}} \quad \Delta \Delta \mathrm{S}_{\mathrm{d}} \\
& \text { Dados ajustados } \\
& -\Delta \mathrm{H}_{\mathrm{d}}=34,197+4,735 \cdot \exp \left(8,313 / \mathrm{X}_{\mathrm{e}}\right) \mathrm{R}^{2}=0,980 \\
& -.-\Delta \mathrm{S}_{\mathrm{d}}=0,094+0,029 \cdot \exp \left(6,791 / \mathrm{X}_{\mathrm{e}}\right) \mathrm{R}^{2}=0,987
\end{aligned}
$$

Figura 3. Efeitos da umidade de equilíbrio $\left(\mathrm{X}_{\mathrm{e}}\right)$ na entalpia $\left(\Delta \mathrm{H}_{\mathrm{d}}\right)$ e entropia $\left(\Delta \mathrm{S}_{\mathrm{d}}\right)$ diferenciais do amido de rizomas do lírio-do-brejo (H. coronarium).

menor quantidade de sítios de adsorção altamente polares na sua superfície e, portanto, disponibiliza menor energia para a interação com as moléculas de água, o que o torna um produto menos higroscópico que os amidos acima referidos.

Os parâmetros do modelo exponencial da Equação (16), com $\mathrm{Y}=\Delta \mathrm{H}_{\mathrm{d}}$ e $\mathrm{X}_{\mathrm{e}}$ entre $5 \mathrm{a} 40 \mathrm{~kg}$ de água $/ 100 \mathrm{~kg}$ de matéria

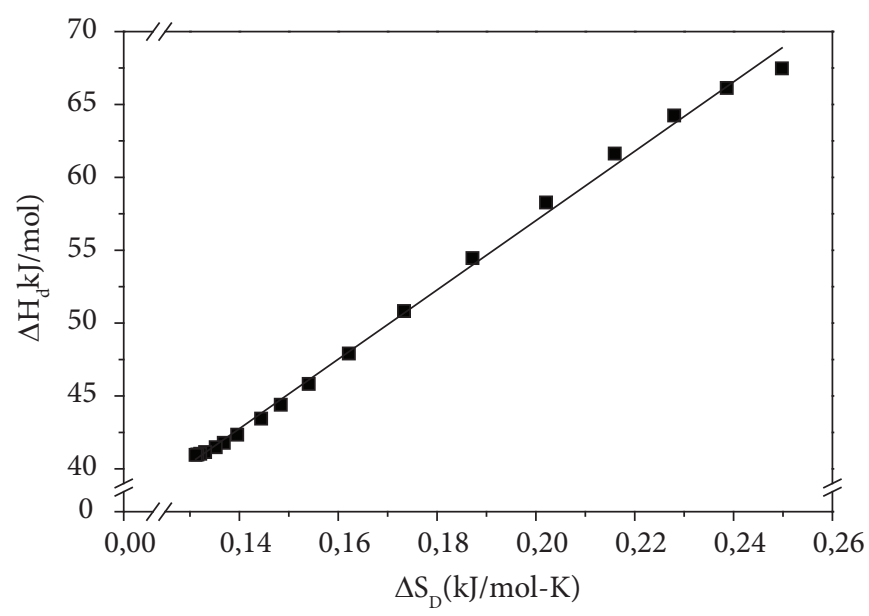

$$
\begin{aligned}
& \text { Dados observados } \\
& \text { - } \\
& \begin{array}{l}
\text { Dados ajustados } \\
\Delta \mathrm{H}_{\mathrm{d}}=9,458+237,877 \Delta \mathrm{S}_{\mathrm{d}} \\
\mathrm{R}^{2}=0,997
\end{array}
\end{aligned}
$$

Figura 4. Correlação entre entalpia diferencial $\left(\Delta \mathrm{H}_{\mathrm{d}}\right)$ e entropia diferencial $\left(\Delta \mathrm{S}_{\mathrm{d}}\right)$ de adsorção de água do amido de rizomas do líriodo-brejo (H. coronarium).

seca, estão na Tabela 4. Este modelo exponencial se ajustou bem aos dados de $\Delta \mathrm{H}_{\mathrm{d}}$, apresentando um $\mathrm{R}^{2}$ de $98 \%$ de acerto e com valor de $\chi^{2}$ próximo a zero, podendo ser utilizado como modelo preditivo.

Mudança no valor de $\Delta \mathrm{S}_{\mathrm{d}}$ se dá de acordo com a quantidade de água que é adsorvida pelo amido. A magnitude dessa mudança varia com a pressão de vapor de água em que a água é adsorvida. O cálculo deste tipo de energia é importante, uma vez que junto à entalpia diferencial de adsorção resulta em energia livre de Gibbs $\left(\Delta \mathrm{G}=\mathrm{AH}_{\mathrm{d}}-\mathrm{T} . \Delta \mathrm{S}_{\mathrm{d}}\right)$ que é uma função termodinâmica de estado que representa a quantidade máxima de energia liberada em um processo ocorrendo a temperatura e pressão constantes que está livre para realizar trabalho útil.

Na Figura 3 constata-se uma forte dependência da $\Delta S_{d}$ com $\mathrm{X}_{\mathrm{e}}$. $\mathrm{O}$ valor de $\Delta \mathrm{S}_{\mathrm{d}}$ diminui com o aumento de $\mathrm{X}_{\mathrm{e}}$, associado à perda da mobilidade das moléculas de água durante o processo de adsorção de água do amido de rizomas do lírio-do-brejo.

Tendências semelhantes foram observadas para amido de batata e amidos com elevados teores de amilose e de amilopectina (AL-MUHTASEB; MCMINN; MAGEE, 2004) e para sementes de sésamo (KAYA; KAHYAOGLU, 2006).

Os parâmetros do modelo exponencial da Equação (16), com $Y=\Delta S_{d}$ e $X_{e}$ entre 5 e $40 \mathrm{~kg}$ de água $/ 100 \mathrm{~kg}$ de matéria seca, também se encontram na Tabela 3 . Os valores de $\mathrm{R}^{2}$ igual a $98,7 \%$ e $\chi^{2}$ próximo a zero indicaram que a Equação 16 pode ser utilizada como modelo preditivo.

Na Figura 4 é mostrada a correlação linear entre a $\Delta \mathrm{H}_{\mathrm{d}} \mathrm{e}$ $\Delta \mathrm{S}_{\mathrm{d}}$, cujo $\mathrm{R}^{2}$ foi próximo a um, indicando que a compensação química entre estes parâmetros termodinâmicos existe. 


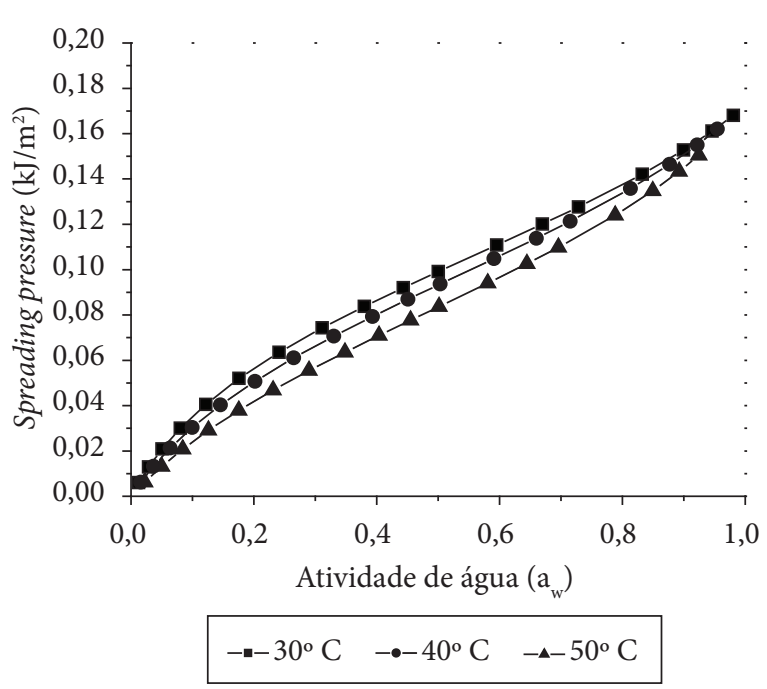

Figura 5. Variação do spreading pressure $(\phi)$ em função da atividade de água $\left(\mathrm{A}_{\mathrm{w}}\right)$ do amido de rizomas do lírio-do-brejo (H. coronarium).

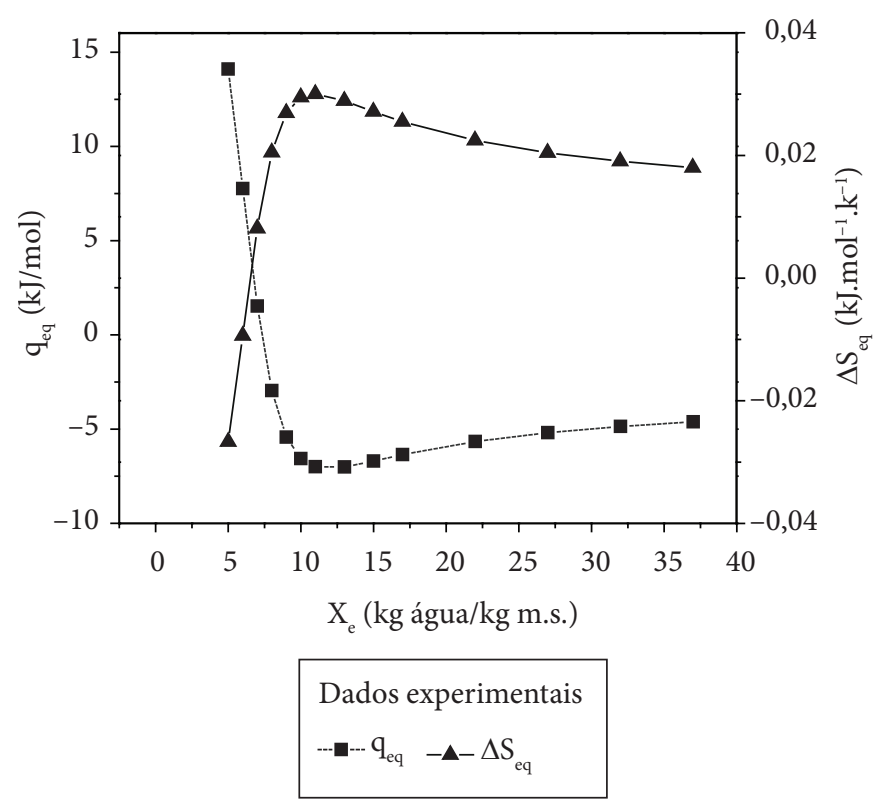

Figura 6. Variação da entalpia $\left(\mathrm{q}_{\mathrm{eq}}\right)$ e entropia $\left(\Delta \mathrm{S}_{\mathrm{eq}}\right)$ integrais em função de $\mathrm{X}_{\mathrm{e}}$ do amido de rizomas do lírio-do-brejo (H. coronarium).

Tabela 4. Parâmetros do modelo exponencial do tipo $\mathrm{Y}=\mathrm{Y}_{0}+\mathrm{a} \cdot \mathrm{e}^{\left(\mathrm{b} / \mathrm{X}_{\mathrm{e}}\right)}$, aplicado às propriedades termodinâmicas do amido de rizomas do lírio-do-brejo (H. coronarium).

\begin{tabular}{crcccc}
\hline Propriedade & \multicolumn{5}{c}{ Parâmetro } \\
\cline { 2 - 6 } termodinâmica & \multicolumn{1}{c}{$\mathrm{Y}_{0}$} & $\mathrm{a}$ & $\mathrm{b}$ & $\mathrm{R}^{2}$ & \multicolumn{1}{c}{$\chi^{2}$} \\
\hline$\Delta \mathrm{H}_{\mathrm{d}}$ & 34,197 & 4,735 & 8,313 & 0,980 & 0,701 \\
$\Delta \mathrm{S}_{\mathrm{d}}$ & 0,094 & 0,029 & 6,791 & 0,987 & $7,80.10^{-6}$ \\
\hline
\end{tabular}

A regressão correspondente ao amido de rizomas do lírio-do-brejo foi (Equação 17):

$\Delta H_{d}=9,458+237,877 . \Delta S_{d} \quad R^{2}=0,997 \quad \chi^{2}=0,277$

Na Equação 17, o coeficiente angular da regressão corresponde à temperatura isocinética das farinhas foi de 237,877 K. Esta temperatura foi menor do que a média harmônica calculada pela Equação 15, resultando um valor de 312,94 K. O $\mathrm{T}_{\mathrm{F}}>\mathrm{T}_{\mathrm{hm}}$ confirma a compensação química linear (KRUG; HUNTER; GRIEGER, 1976) entre a entalpia e a entropia de adsorção de água do amido de rizomas do lírio-do-brejo.

\subsection{Entalpia e entropia integrais de adsorção}

A variação de $\varphi$ em função de $\mathrm{A}_{\mathrm{w}}$ para 30 a $50{ }^{\circ} \mathrm{C}$ do amido de rizomas do lírio-do-brejo está na Figura 5. O $\varphi$ aumenta com o aumento de $\mathrm{A}_{\mathrm{w}}$ e diminui com o aumento da temperatura, embora se observe elevação a partir de $A_{w}=0,9$, indicando a dissolução endotérmica do material farináceo.

Os valores de $\mathrm{q}_{\mathrm{eq}}$ foram obtidos para cada valor de $\phi$. Os resultados estão na Figura 6, na qual se mostra a dependência aos valores de $\mathrm{X}_{\mathrm{e}}$.

Este tipo de energia é atribuído aos sítios primários de adsorção de água altamente polares presentes na superfície do material (FASINA; AJIBOLA; TYLER, 1999). Quando em valores de $\mathrm{X}_{\mathrm{e}}$ muito baixos (em torno de $5 \mathrm{~kg}$ água/100 kg m.s.) moléculas de água são adsorvidas formando uma monocamada sobre os sítios de adsorção e o sistema de adsorção ganha energia (aproximadamente de 14,1 kJ/mol). Após formação da monocamada, $\mathrm{q}_{\mathrm{eq}}$ diminui à medida que os sítios de adsorção são ocupados por ligações relativamente fracas de moléculas de água formando camadas sucessivas (KAYA; KAHYAOGLU, 2006). O menor valor desta energia foi observado em $X_{e}=11 \mathrm{~kg}$ água $/ 100 \mathrm{~kg}$ m.s. correspondendo a $\mathrm{q}_{\mathrm{eq}}=-6,99 \mathrm{~kJ} / \mathrm{mol}$, respectivamente, diminuindo em torno de $21 \mathrm{~kJ} / \mathrm{mol}$.

Os resultados da $\Delta \mathrm{S}_{\text {eq }}$ também estão esquematizados em forma de gráficos na Figura 6 . Ao contrário de $\mathrm{q}_{\mathrm{eq}}, \Delta \mathrm{S}_{\mathrm{eq}}$ experimenta um aumento em função de $\mathrm{X}_{\mathrm{e}}$, isto é, esta propriedade termodinâmica aumenta exponencialmente por acréscimo do teor de $\mathrm{X}_{\mathrm{e}}$.

Vários pesquisadores (AVIARA; AJIBOLA; DAIRO, 2002; KAYA; KAHYAOGLU, 2006) sugeriram que a diminuição de $\Delta \mathrm{S}_{\text {eq }}$ com a diminuição de $\mathrm{X}_{\mathrm{e}}$ é atribuída à diminuição do movimento das moléculas de água à medida que os sítios de adsorção disponíveis forem saturados. O mínimo valor de $\Delta \mathrm{S}_{\mathrm{eq}}$ foi registrado quando a água adsorvida saturou completamente a monocamada $\left(\mathrm{X}_{\mathrm{e}}=5 \mathrm{~kg}\right.$ água $/ 100 \mathrm{~kg} \mathrm{~m} . \mathrm{s}$. $)$ sendo este de $-0,03 \mathrm{~kJ} / \mathrm{mol}-\mathrm{K}$. O aumento subsequente de $\Delta \mathrm{S}_{\text {eq }}$ disponibiliza a umidade em forma de água livre formando a multicamada, verificando-se este em $\mathrm{X}_{\mathrm{e}}=11 \mathrm{~kg}$ água/100 kg m.s. com $\Delta \mathrm{S}_{\text {eq }}=0,03 \mathrm{~kJ} / \mathrm{mol}-\mathrm{K}$.

\section{Conclusões}

As isotermas demonstraram que quanto maior a temperatura de adsorção, entre 30 e $50^{\circ} \mathrm{C}$ e em atividades de água > 5,0, maior a adsorção de água do amido de rizomas do lírio-do-brejo pela 
presença de grânulos de amido danificados. A Equação de GAB se ajustou bem às isotermas, apresentando coeficientes de correlação $\left(\mathrm{R}^{2}\right)$ próximos à unidade e testes de $\chi^{2}$ próximos a zero.

A entalpia diferencial diminui exponencialmente com o aumento da umidade de equilíbrio, aproximando-se do valor do calor latente de vaporização da água pura em umidade de $11 \mathrm{~kg}$ de água por $100 \mathrm{~kg}$ de amostra seca.

Em menores valores de umidades de equilíbrio o amido de rizomas do lírio-do-brejo mostrou baixo valor de entalpia diferencial de $58 \mathrm{~kJ} / \mathrm{mol}$ o que sugere que o amido de rizomas do lírio-do-brejo possui baixa quantidade de sítios de adsorção altamente polares disponíveis para a interação com moléculas de água, tornando-se produto pouco higroscópico.

A entropia diferencial diminui com o aumento da umidade de equilíbrio, apresentando um valor máximo de $0,20 \mathrm{~kJ} / \mathrm{mol}-\mathrm{K}$, que foi atingido em baixos valores de umidade de equilíbrio.

A temperatura isocinética de $237,9 \mathrm{~K}$ confirmou a compensação química linear entre a entalpia e entropia diferenciais do amido de rizomas do lírio-do-brejo.

A entalpia integral e a entropia integral aumentaram continuamente com o teor de umidade de equilíbrio, porém com valores negativos para a entropia integral. As energias nas proximidades da formação da monocamada e da multicamada destas propriedades termodinâmicas integrais foram de $14,10 \mathrm{~kJ} / \mathrm{mol} \mathrm{e}-0,03 \mathrm{~kJ} / \mathrm{mol}-\mathrm{K}$ e de $-6,99 \mathrm{~kJ} / \mathrm{mol}$ e $0,03 \mathrm{~kJ} / \mathrm{mo}-\mathrm{K}$ para $\mathrm{q}_{\mathrm{eq}}$ e $\Delta \mathrm{S}_{\mathrm{eq}}$, respectivamente.

\section{Agradecimentos}

À Embrapa Agroindústria de Alimentos do Rio de Janeiro (CTAA/EMBRAPA - RJ), à Universidade Estadual de Goiás, pelo suporte técnico, e à fazenda Mata Taquaral por ter cedido a matéria-prima utilizada.

\section{Referências bibliográficas}

AGAREZ, F. V.; PEREIRA, C.; ATÍDIO, C. Botânica: Taxionomia, morfologia e reprodução dos angiospermae: chaves para determinação das famílias. 2 ed. Rio de Janeiro: Âmbito Cultural, 1994. 233 p.

AGUERRE, R. J.; SUAREZ, C.; VIOLLAZ, P. E. Enthalpy entropy compensation in sorption phenomena: application to the prediction of the effect of temperature on food isotherms. Journal of Food Science, v. 51, n. 6, p. 1547-1549, 1986.

AL-MUHTASEB, A. H.; MCMINN, W. A. M.; MAGEE T. R. A. Water sorption isotherms of starch powders. Part 2: Thermodynamic characteristics. Journal of Food Engineering, v. 62, n. 2, p. 135-142, 2004.

ASSOCIATION OF OFFICIAL ANALYTICAL CHEMISTS - AOAC. Official methods of analysis. 19 ed. Washington, 2000.

ASCHERI, D. P. R. et al. Obtenção de farinhas mistas pré-gelatinizadas a partir de arroz e bagaço de jabuticaba: efeito das variáveis de extrusão nas propriedades de pasta. Boletim CEPPA, v. 24, n. 1, p. 115-144, 2006.

ASCHERI, D. P. R.; NASCIMENTO, G. C.; ASCHERI, J. L. R. Características de adsorción de agua de la harina de arroz soluble a varias temperaturas. Alimentaria, v. 40, n. 349, p. 111-119, 2003.
AVIARA, N. A.; AJIBOLA, O. O.; DAIRO, U. O. Thermodynamics of moisture sorption in sesame seed. Biosystems Engineering, v. 83, n. 4, p. 423-431, 2002.

AVIARA, N. A.; AJIBOLA, O. O.; ONI, S. A. Sorption equilibrium and thermodynamic characteristics of soya bean. Biosystems Engineering, v. 87, n. 2, p. 179-190, 2004.

BELL, L. N.; LABUZA, T. P. Moisture sorption - Practical aspects of isotherm measurement and use. 2 ed. St. Paul: American Association of Cereal Chemists, Inc., 2000. 122 p.

BENADO, A. L.; RIZVI, S. S. H. Thermodynamic properties of water in rice as calculated from reversible and irreversible isotherms. Journal of Food Science, v. 50, n. 2, p. 101-105, 1985.

BRASIL. Decreto n. 12.486, de 20 de outubro de 1978. Normas técnicas especiais relativas a alimentos e bebidas. Diário Oficial do Estado de São Paulo, São Paulo, 21 de outubro de 1978. p. 20.

DEMIATE, I. M.; OETTERER, M.; WOSIACKI, G. Characterization of Chestnut (Castanea sativa, Mill) Starch for Industrial Utilization. Brazilian Archives of Biology and Technology, v. 44, n. 1, p. 69-78, 2001.

FASINA, O. O. Thermodynamic properties of sweet potato. Journal of Food Engineering, v. 75, n. 2, p. 149-155, 2006.

FASINA, O.; AJIBOLA, O.; TYLER, R. Thermodynamics of moisture sorption in winged bean seed and gari. Journal of Food Process Engineering, v. 22, n. 6, p. 405-418, 1999.

FASINA, O.; SOKHANSANJ, S.; TYLER, R. Thermodynamics of moisture sorption in alfalfa pellets. Drying Technology, v. 15, n. 5, p. 1553-1570, 1997.

FERREIRA, C. D.; PENA, R. S. Comportamento higroscópico da farinha de pupunha (Bactris gasipaes). Ciência e Tecnologia de Alimentos, v. 23, n. 2, p. 251-255, 2003.

GOMES, F. P. Curso de Estatística Experimental. 14 ed. Piracicaba: ESALQ/USP, 2000. 477 p.

GREENSPAN, L. Humidity fixed points of binary saturated aqueous solutions. Journal of Research o the National Bureau of Stand, v. 81A, n. 1, p. 89-96, 1977.

HILL, P. E.; RIZVI, S. S. H. Thermodynamic parameters and storage stability of drum dried peanut flakes. Lebensmittel-Wissenschaft und Technologie, v. 15, n. 2, p. 185-190, 1982.

HOOVER, R.; SINNOTT, A. W.; PERERA, C. Physicochemical characterization of starches from amaranthus cruentus grains. Starch/stärke, v. 50, n. 11-12, p. 456-463, 1998.

HOOVER, R. Composition, molecular structure, and physicochemical properties of tuber and root starches: a review. Carbohydrate Polymers, v. 45, n. 3, p. 253-267, 2001.

HOSSAIN, M. D. et al. Sorption isotherms and heat of sorption of pineapple. Journal of Food Engineering, v. 48, n. 2, p. 103-107, 2001.

IGLESIAS, H. A.; CHIRIFE, J.; VIOLLAZ, P. Thermodynamics of water vapour sorption by sugar beet root. Journal of Food Technology, v. 11, n. 1, p. 91-101, 1976.

KAYA, S.; KAHYAOGLU, T. Influence of dehulling and roasting process on the thermodynamics of moisture adsorption in sesame seed. Journal of Food Engineering, v. 76, n. 2, p. 139-147, 2006.

KISSMANN, K. G, GROTH, D. Plantas infestantes e nocivas. São Paulo: Basf Brasileira, 1991. p. 590-593.

KRUG, R. R.; HUNTER, W. G.; GRIEGER, R. A. Enthalpy-entropy compensation. 1 - Some fundamental statistical problems associated with the analysis of Van't Hoff and Arrhenius data. Journal Physical Chemistry, v. 80, n. 21, p. 2335-2341, 1976. 
LEONEL, M. et al. Extração e caracterização de amido de Biri (Canna edulis). Brazilian Journal of food technology, v. 5, n. 77, p. 27-32, 2002.

LI, R. et al. Equilibrium moisture content of cellulosic materials at high temperature. Drying Technology, v. 12, n. 4, p. 823-847, 1994.

MACEDO, J. F. O gênero Hedychium koening (Zingiberaceae) no Estado de Minas Gerais. Daphne, v. 7, n. 2, p. 27-31, 1997.

MADAMBA, P. S.; DRISCOLL, R. H.; BUCKLE, K. A. Enthalpy-entropy compensation models for sorption and browning of garlic. Journal of Food Engineering, v. 28, n. 2, p. 109-119, 1996.

MAZZA, G. Thermodynamic considerations of water vapour sorption by horseradish roots. Lebensmittel-Wissenschaft und Technologie, v. 13, p. $13-17,1980$.

MCMINN, W. A. M.; MAGEE, T. R. A. Thermodynamic properties of moisture sorption of potato. Journal of Food Engineering, v. 60, n. 2, p. 157-165, 2003.

MESSE, J. Y. ThermExcel. Issy Les Moulineaux: n. 45188030600019, 2003. Disponível em: <http://www.thermexcel.com/english/index. htm>. Acesso em: 23 Jun. 2007.

PALIPANE, K. B.; DRISCOLL, R. H. Moisture sorption characteristics of in-shell macadamia nuts. Journal of Food Engineering, v. 18, n. 1, p. 63-76, 1992.

REYES, F. G. R. et al. Characterization of starch from ginger root (Zingiber officinale). Starch/stärke, v. 34, n. 2, p. 40-44, 1982.
RIZVI, S. S. H. Thermodynamic properties of food in dehydration. In: RAO, M. A.; RIZVI, S. S. H. Engineering properties of foods. New York: Marcel Dekker Inc, 1986. p. 223-309.

ROMERO, J. T. et al. Enthalpy-entropy compensation based on isotherms of mango. Ciência e Tecnologia de Alimentos, v. 25, n. 2, p. 297-303, 2005.

SANTOS, S. B.; PEDRALLI, G.; MEYER, S. T. Aspectos da fenologia e ecologia de Hedychium coronarium (Zingiberaceae) na estação ecológica do Tripuí, Ouro Preto-MG. Planta Daninha, v. 23, n. 2, p. 175-180, 2005.

SINGH, N. et al. Physicochemical, thermal, morphological and pasting properties of starches from some indian black gram (phaseolus mungo 1.) cultivars. Starch/stärke, v. 56, n. 11, p. 535-544, 2004.

STAMM, A. J. Wood and cellulose science. New York: The Ronald Press, 1964. 549 p.

TSAMI, E. et al. Heat of sorption of water in dried fruits. International Journal Food Science and Technology, v. 25, n. 3, p. 350-359, 1990.

VERWIMP, T. et al. Isolation and characterization of rye starch. Journal of cereal science, v. 39, n. 11, p. 85-90, 2004.

WILLIAMS, P. C.; KUZINA, F. D.; HLYNKA, I. A rapid colorimetric method for estimating the amylose content of starches and flours. Cereal Chemistry, v. 47, n. 4, p. 411-420, 1970. 